\title{
Rasgos Residuales de Dislexia en Estudiantes Preuniversitarios
}

Dyslexia persistent symptoms in pre-university students

Traços residuais da dislexia em estudantes universitários

Fabiola R. Gómez-Velázquez

Universidad de Guadalajara, Instituto de Neurociencias, México fabiola.gomez@academicos.udg.mx https://orcid.org/0000-0002-2081-6280

Alicia Martínez-Ramos

Universidad de Guadalajara, Departamento de Neurociencias, México martinez.ramos.alicia@gmail.com https://orcid.org/0000-0002-8136-330X

Itzel Vergara

Universidad de Guadalajara, Instituto de Neurociencias, México itzel.vergara@ymail.com https://orcid.org/0000-0002-3181-3376

Vanessa D. Ruiz-Stovel Universidad de Guadalajara, Instituto de Neurociencias, México vanessa.ruizstovel@academicos.udg.mx https://orcid.org/0000-0003-0324-0643

Jacobo J. Brofman-Epelbaum Universidad Panamericana, Campus Guadalajara, México jbrofman@up.edu.mx https://orcid.org/0000-0001-6851-1163 


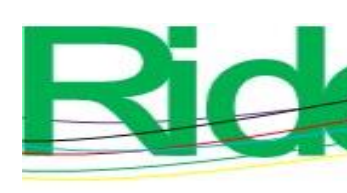

Revista Iberoamericana para la
Investigación y el Desarrollo Educativo
ISSN $2007-7467$

\section{Resumen}

Los rasgos de dislexia tienden a persistir hasta la edad adulta, desafortunadamente muchos jóvenes nunca son diagnosticados y continúan lidiando con sus dificultades en etapas preuniversitarias. En el presente estudio se buscó identificar las dificultades en la vida cotidiana que reportan jóvenes preuniversitarios en un cuestionario de dislexia para adultos, para lo cual se compararon las respuestas de 52 estudiantes del último grado de bachillerato, entre 17 y 18 años de edad, 26 jóvenes clasificados como disléxicos por su baja velocidad lectora y pobre conocimiento ortográfico y 26 sin dificultades lectoras, se analizó cada pregunta y su relación con el rendimiento lector. Los resultados mostraron que los jóvenes con dislexia no reportan problemas con la rotación de números, pero sí con el llenado de formas, la ortografía y la lectura en voz alta, en cambio los jóvenes sin dificultades lectoras señalaron con mayor frecuencia tener problemas para discriminar derecha-izquierda, leer un mapa o recordar lo que acaban de leer, aspectos que muchas veces se han relacionado con dislexia. Estos resultados confirman que muchas creencias populares sobre la dislexia no tienen un fundamento científico que las sustente y que los puntajes totales de los cuestionarios de dislexia podría señalar erróneamente a personas sin dificultades lectoras. Proponemos un cuestionario breve enfocado a explorar sólo siete aspectos específicos de la lectura que ayudarían a indentificar rasgos residuales de dislexia en jóvenes, con el fin de brindarles apoyo para continuar sus estudios académicos.

Palabras clave: cuestionario de dislexia, problemas lectores, jóvenes, lecto-escritura, ortografía.

\section{Abstract}

The symptoms of dyslexia tend to persist into adulthood. Unfortunately, many young people with reading difficulties are never diagnosed and continue dealing with their reading disabilities in postsecondary stages. The objective of this study was to identify residual problems in pre-university students through the application of a dyslexia questionnaire. Fiftytwo students of the last year of high school participated, between 17 and 18 years, 26 young people classified as dyslexic because of poor reading performance and poor spelling, and 26 young people without reading difficulties. Dyslexic teenagers do not have number rotation problems, but they reported problems filling out forms, spelling correctly, and reading aloud. On the other hand, teenagers without dyslexia more often pointed out having problems with 


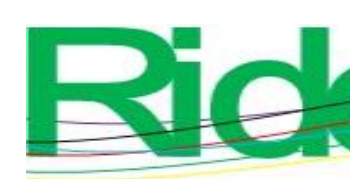

\section{Revista Iberoamericana para la Investigación y el Desarrollo Educativo ISSN $2007-7467$}

left-right discrimination, reading maps, or remembering what they had read, aspects that have often been related to dyslexia. Results corroborate that many popular beliefs about dyslexia are not based on scientific evidence and that most questionnaires could erroneously signalize people without reading difficulties. We propose a short questionnaire focused on exploring only seven specific aspects of reading that would help identify dyslexia persistent symptoms in young adults to support them to continue their academic studies.

Keywords: dyslexia checklist, reading disabilities, teenagers, orthographic knowledge, spelling.

\section{Resumo}

Os traços da dislexia tendem a persistir na idade adulta, infelizmente muitos jovens nunca são diagnosticados e continuam a lidar com suas dificuldades na fase pré-universitária. No presente estudo, buscou-se identificar as dificuldades no cotidiano relatadas por jovens préuniversitários em um questionário de dislexia para adultos, para o qual foram respondidas 52 estudantes do último ano do ensino médio, entre 17 e 18 anos de idade., foram comparados 26 jovens classificados como disléxicos devido à baixa velocidade de leitura e baixo conhecimento ortográfico e 26 sem dificuldades de leitura, foram analisadas cada questão e sua relação com o desempenho na leitura. Os resultados mostraram que os jovens com dislexia não relatam problemas com rotação de números, mas com preenchimento de formulários, ortografia e leitura em voz alta, enquanto jovens sem dificuldades de leitura relataram com maior frequência ter problemas para discriminar direita-esquerda, ler mapa ou lembrar o que faziam. acabei de ler, aspectos que muitas vezes têm sido relacionados à dislexia. Esses resultados confirmam que muitas crenças populares sobre a dislexia não têm base científica para apoiá-las e que os escores totais dos questionários de dislexia podem apontar erroneamente para pessoas sem dificuldades de leitura. Propomos um pequeno questionário focado em explorar apenas sete aspectos específicos da leitura que ajudariam a identificar traços residuais de dislexia em jovens, a fim de fornecer-lhes suporte para continuar seus estudos acadêmicos.

Palavras-chave: questionário de dislexia, problemas de leitura, jovens, alfabetização, ortografia. 


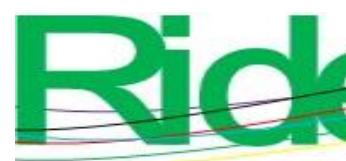

Fecha Recepción: Abril 2021
Revista Iberoamericana para la

Investigación y el Desarrollo Educativo ISSN $2007-7467$

\section{Introducción}

Las dificultades en el aprendizaje de la lectura o dislexia del desarrollo representan un problema frecuente en la etapa escolar. Estas dificultades se derivan de un trastorno del neurodesarrollo que es independiente de las capacidades cognitivas, del ambiente sociocultural o de las oportunidades de aprendizaje que haya tenido el individuo, sus manifestaciones tienden a persistir hasta la etapa adulta, a pesar de que el individuo se haya expuesto repetidamente a actividades de lectura y escritura.

Los jóvenes que arrastran dificultades lectoras desde la infancia se enfrentan con demandas crecientes de lectura como: abstraer información a partir de textos de manera cada vez más rápida con el objeto de hacer resúmenes; escribir ensayos o reportes de manera clara, creativa y sin cometer errores ortográficos; todo lo cual representa un gran reto para su adaptación escolar. Al impacto académico, se le añade el impacto emocional y social que tienen las dificultades lectoras sobre el desarrollo. Muchos de estos jóvenes presentan baja autoestima y pobre interés por los logros académicos, todo lo cual puede incluso llevarlos a la deserción escolar.

Se ha reportado, por ejemplo, que una mayor proporción de jóvenes con pobre conocimiento ortográfico y bajo rendimiento lector no continúan estudiando después del bachillerato, en comparación con aquéllos jóvenes con un conocimiento ortográfico y una lectura normales (González-Garrido, et al., 2014). La mayoría de ellos no tienen un diagnóstico de dificultades lectoras, debido principalmente a la falta de consenso en el ambito académico sobre los rasgos distintivos de la dislexia en el adulto y a la falta de instrumentos diagnósticos fiables y de aplicación rápida, que permitan detectar tempranamente el problema. La falta de detección en etapas tempranas limita mucho las posibilidades de que los jóvenes reciban una atención oportuna y adecuada a sus dificultades.

En los años recientes se ha incrementado el estudio de las dificultades lectoras en adultos, motivado por el interés en identificar cuáles son las principales dificultades que persisten en las personas con dislexia (Soriano-Ferrer y Piedra-Martínez, 2016). Uno de los métodos más empleados para identificar esas dificultades es la aplicación de cuestionarios o escalas de autoreporte, que exploran aspectos tan diversos como: dificultades visoperceptuales, de atención, de orientación en el espacio, de lenguaje, de escritura y, por supuesto, de lectura. 


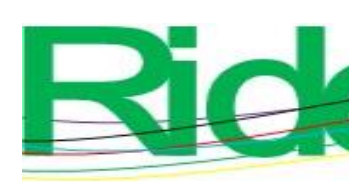

Revista Iberoamericana para la
Investigación y el Desarrollo Educativo
ISSN $2007-7467$

su muestra sólo incluyeron 8 mujeres y 4 hombres universitarios con diagnóstico de dislexia, con un rango muy amplio de edad, entre 20 y 49 años.

En adolescentes hablantes del español, se ha encontrado que un bajo conocimiento ortográfico se relaciona con lentitud y baja eficiencia lectora, lo cual además se ha asociado con menor modulación y lateralización en la respuesta electrofisiológica ante el procesamiento de errores ortográficos, cuando se compara con jóvenes con altas habilidades ortograficas (González-Garrido et al., 2014), además de que se ha relacionado con el reclutamiento neural de un mayor número de áreas cerebrales para el procesamiento de palabras, como una posible estrategia compensatoria de los jóvenes con pobre conocimiento ortográfico (González-Garrido et al., 2017).

Dados los hallazgos provenientes de ortografías transparentes que consideran que los rasgos residuales más importantes de la dislexia en el adulto son la lentitud para leer y el pobre conocimiento ortográfico, decidimos explorar, en un grupo de jóvenes con estas características, las respuestas a una escala de autoreporte con el objetivo principal de identificar los aspectos que ellos perciben como deficitarios en su vida cotidiana y determinar qué items son más sensibles para la identificación de jóvenes preuniversitarios que enfrentan problemas lectores en una etapa crítica para su desarrollo académico.

\section{Materiales y método}

Se estudió un grupo de 52 estudiantes del último año de bachillerato, diestros, entre 17 y 18 años de edad, con el español como lengua materna, divididos en dos grupos: Controles y Disléxicos. En el grupo de Disléxicos se incluyó a los jóvenes que presentaron los dos rasgos considerados como déficits residuales de la dislexia en el adulto, tenían una lectura significativamente más lenta de lo esperado para su edad y grado académico, además de un pobre conocimiento ortográfico.

- Disléxicos: 26 jóvenes (15 hombres y 11 mujeres) con un total de errores homófonos $\geq$ percentil 85, según los baremos de la Batería de Conocimiento Ortográfico (BCO, Gómez-Velázquez et al., 2014) y una velocidad lectora $\leq$ al percentil 16 según normas de la Evaluación Neuropsicológica Infantil (ENI, Matute et al., 2007) para jóvenes de 16 años (en México no existen normas de velocidad lectora para mayores de 16 años).

- Controles: 26 jóvenes (10 hombres y 16 mujeres) con un nivel de errores en la BCO $\leq$ al percentil 70 y una velocidad lectora $\geq$ percentil 35 según normas de la ENI para jóvenes de 16 años. 


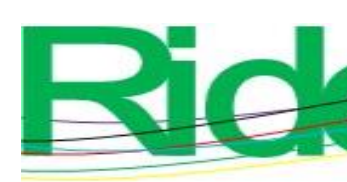

\section{Revista lberoamericana para la Investigación y el Desarrollo Educativo ISSN $2007-7467$}

Se compararon los resultados de edad, CI, conocimiento ortográfico y rendimiento lector entre los grupos, usando una prueba t de Student para grupos independientes, así como en las respuestas en la escala de Dislexia (usando la prueba Levene para hacer correcciones cuando se violó el principio de igualdad de las varianzas). Adicionalmente se hicieron correlaciones de Pearson para determiner las posibles relaciones entre las variables.

El presente Proyecto fue realizado de acuerdo con los principios de la Declaración de Helsinki y fue aprobado por el comité de ética del Instituto de Neurociencias. Todos los participantes (o sus padres en el caso de menores de edad) firmaron una carta de consentimiento informado de su participación en la investigación.

\section{Resultados}

Las características generales de la muestra de estudio se presentan en la Tabla 1. No se encontraron diferencias significativas entre los grupos en la edad o el CI estimado. Como era de esperarse, dado que así se seleccionó a la muestra, los jóvenes del grupo de Disléxicos presentaron un significativo menor nivel de conocimiento ortográfico y menor velocidad lectora en comparación con los jóvenes del grupo Control. Los resultados de la evaluación de lectura mostraron además que los jóvenes del grupo de Disléxicos presentaron también problemas con la eficiencia al leer, es decir, cometieron más errores no corregidos durante su lectura en voz alta, aunque la comprensión lectora se encontraba conservada.

Para analizar los resultados de las respuestas de los jóvenes en el Cuestionario Revisado de Dislexia en el Adulto (20 preguntas), en primer lugar, se hizo un análisis general sumando el total de respuestas afirmativas de cada participante y se compararon las medias grupales, no se hallaron diferencias significativas entre los jóvenes del grupo control y los jóvenes con dislexia $\left(\mathrm{t}_{(50)}=-1.134, \mathrm{p}=2.62\right.$ : media del grupo Control $=4.85, \mathrm{DS}=2.1$; media de Disléxicos = 5.6, DS = 2.7). 


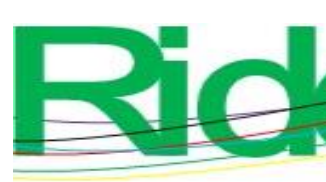

Revista Iberoamericana para la Investigación y el Desarrollo Educativo ISSN 2007 - 7467

Tabla 1. Caracterización de la muestra

\begin{tabular}{|c|c|c|c|c|c|}
\hline & Grupo & Media & $\begin{array}{c}\text { Desviación } \\
\text { estándar }\end{array}$ & $\mathrm{t}(\mathrm{gl})$ & $\mathrm{p}$ \\
\hline \multirow{2}{*}{ Edad } & Controles & 17.6 & 0.58 & \multirow{2}{*}{$-1.672(44.6)$} & \multirow{2}{*}{0.102} \\
\hline & Disléxicos & 17.8 & 0.40 & & \\
\hline \multirow{2}{*}{ CI Estimado ${ }^{\mathrm{a}}$} & Controles & 104.0 & 10.4 & \multirow{2}{*}{$1.181(50.0)$} & \multirow{2}{*}{0.243} \\
\hline & Disléxicos & 101.1 & 7.1 & & \\
\hline \multirow{2}{*}{$\begin{array}{l}\text { Conocimiento } \\
\text { Ortográfico }^{b}\end{array}$} & Controles & 13.81 & 6.6 & \multirow{2}{*}{$\begin{array}{c}-14.699 \\
(31.6)\end{array}$} & \multirow{2}{*}{0.000} \\
\hline & Disléxicos & 34.19 & 2.4 & & \\
\hline \multirow{2}{*}{ Velocidad lectora $^{\mathrm{c}}$} & Controles & 161.1 & 8.6 & \multirow{2}{*}{$\begin{array}{l}13.934 \\
(50.0)\end{array}$} & \multirow{2}{*}{0.000} \\
\hline & Disléxicos & 124.5 & 10.2 & & \\
\hline \multirow{2}{*}{ Errores al leer } & Controles & 3.2 & 2.9 & \multirow{2}{*}{$-4.074(38.0)$} & \multirow{2}{*}{0.000} \\
\hline & Disléxicos & 8.1 & 5.4 & & \\
\hline \multirow{2}{*}{$\begin{array}{l}\text { Comprensión } \\
\text { lectora }\end{array}$} & Controles & 6.6 & 2.4 & \multirow{2}{*}{$1.373(50.0)$} & \multirow{2}{*}{0.176} \\
\hline & Disléxicos & 5.7 & 2.3 & & \\
\hline
\end{tabular}

${ }^{\text {a }}$ Cociente intelectual estimado a partir de las subescalas de Vocabulario y Diseño con

Cubos del WAIS-III. ${ }^{\mathbf{b}}$ Media del total de errores homófonos (sustitución de grafías homófonas como B-V, S-C-Z, G-J, Y- LL u omisión de H) en 4 tareas de la Bateria de Conocimiento Ortográfico BCO). ${ }^{\mathbf{c}}$ Media de palabras leídas por minuto en voz alta en un texto expositivo.

Se compararon los resultados entre hombres y mujeres (en el presente estudio no se buscó una distribución equitativa de mujeres y hombres por la ya conocida mayor prevalencia de la dislexia en hombres), pero dado que la distribución por sexo en cada grupo era diferente, las diferencias se analizaron intragrupo. En el grupo Control no se hallaron diferencias significativas entre hombres y mujeres en la cantidad de respuestas afirmativas al cuestionario, pero en el grupo de Disléxicos se encontró que una mayor proporción de mujeres reportó problemas para hablar en público $\left(\mathrm{t}_{(10)}=2.887, \mathrm{p}<0.05\right)$ y para el cálculo mental $\left(\mathrm{t}_{(24)}=2.422, \mathrm{p}<0.05\right)$, en comparación con los hombres.

En segundo lugar, se hizo un análisis de las respuestas afirmativas a cada pregunta, comparando los dos grupo (Figura 1). Se encontraron diferencias significativas entre los grupos sólo en tres preguntas: una mayor proporción de jóvenes Disléxicos reportaron tener dificultades con el llenado de formas $\left(\mathrm{t}_{(35)}=-2.076, \mathrm{p}=0.043\right)$, con la ortografía $\left(\mathrm{t}_{(50)}=\right.$ $3.305, \mathrm{p}=0.002)$ y con la lectura en voz alta $\left(\mathrm{t}_{(46)}=-2.200, \mathrm{p}=0.032\right)$, en comparación con 


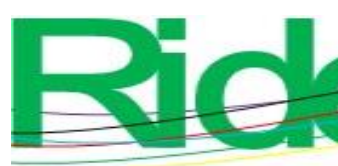

\section{Revista Iberoamericana para la Investigación y el Desarrollo Educativo ISSN $2007-7467$}

cuestionario, los jóvenes presentaron mayor número de errores al leer $(\mathrm{r}=0.388, \mathrm{p}<0.01)$, pero sin relación con otros procesos lectores como la velocidad y la comprensión o con el CI estimado.

Dada esta baja relación del puntaje total del RADC con el proceso lector, se procedió a analizar la relación de cada item con el puntaje total. Se observó que un número alto de respuestas afirmativas al cuestionario, no necesariamente indica la presencia de dificultades con la lecto-escritura sino que podría indicar problemas en otros dominios, ya que se encontraron altas correlaciones $(\mathrm{p}<0.01)$ entre el puntaje total y la frecuencia de respuestas afirmativas a problemas tan diversos como: la mala ortografía $(\mathrm{r}=0.396)$; tomar y pasar mensajes de teléfono $(\mathrm{r}=0.489)$; hacer sumas en la mente $(\mathrm{r}=0.415)$; cambiar el orden de números de teléfono $(\mathrm{r}=0.442)$; confundir citas $(\mathrm{r}=0.464)$; llenar formas o aplicaciones $(\mathrm{r}$ $=0.452)$; $\mathrm{y}$ aprender las tablas de multiplicar $(\mathrm{r}=0.425)$. También se encontró una correlación menor, pero significativa $(\mathrm{p}<0.05)$, entre el total del RADC con la incidencia de dificultades como: decir en orden exacto los sonidos de palabras largas $(r=0.276)$ y decir los meses del año en orden inverso $(r=0.330)$.

Vinegrad (1994), propuso que un total de 9 o más respuestas afirmativas al cuestionario es un poderoso indicador de dificultades lectoras. En nuestros resultados, sólo 4 jóvenes tuvieron 9 o más respuestas afirmativas, tres disléxicos y un control. No fue posible agrupar al resto de los jóvenes de acuerdo con el número total de respuestas al cuestionario.

Por otra parte, se analizó la relación entre el CI estimado con el proceso lector y las respuestas al cuestionario. Los resultados mostraron que los jóvenes con mayor CI presentaron mayores puntajes de comprensión lectora $(\mathrm{r}=0.368, \mathrm{p}<0.01)$, pero no se encontraron correlaciones significativas del CI con otras variables lectoras o con las respuestas al cuestionario de dislexia.

Adicionalmente, se analizó la relación entre las variables lectoras con cada preguntas del cuestionario, encontrando que: un mayor número de errores de ortografía en la BCO correlacionó significativamente con una mayor proporción de jóvenes que reportaron que les desagrada leer en voz alta $(\mathrm{r}=0.342, \mathrm{p}<0.01)$ y que reportaron tener mala ortografía $(\mathrm{r}=$ 0.493, p < 0.01); del mismo modo, una menor velocidad lectora se relacionó con los jóvenes que señalaron que les desagrada leer en voz alta $(r=-0.276, p<0.05)$, tienen mala ortografía $(\mathrm{r}=-0.375, \mathrm{p}<0.01)$ y además tienen dificultades para llenar formas $(\mathrm{r}=-0.311, \mathrm{p}<0.05)$; por otra parte, un mayor número de errores de lectura se relacionó con mayor proporción de personas que aceptaron que les desagrada leer en voz alta $(r=0.406, p<0.01)$, les toma más 


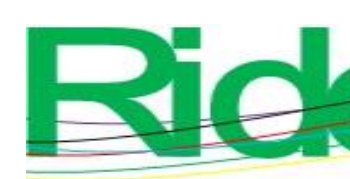

Revista Iberoamericana para la
Investigación y el Desarrollo Educativo
ISSN $2007-7467$

tiempo leer una página $(\mathrm{r}=0.351, \mathrm{p}<0.05)$, les desagrada leer libros gruesos $(\mathrm{r}=0.299, \mathrm{p}<$ 0.05) y tienen mala ortografía $(\mathrm{r}=0.443, \mathrm{p}<0.01)$; finalmente, una baja comprensión lectora sólo se relacionó con mayor proporción de jóvenes que indicaron que les desagrada leer libros gruesos $(r=-0.394, p<0.01)$.

\section{Discusión}

El principal interés de la presente investigación fue determinar si las respuestas a una escala autoaplicable diseñada para detectar dislexia en adultos (RADC, Vinegrad, 1994), tienen efectivamente relación con las dificultades lectoras residuales en jóvenes preuniversitarios.

Los jóvenes de la presente investigación, no tenían un diagnóstico previo de dislexia, sin embargo, al momento de la selección exhibieron importantes dificultades con dos aspectos que se consideran en la literatura como rasgos residuales de dislexia en el adulto: pobre conocimiento ortográfico y lenta velocidad lectora. Los jóvenes del grupo de disléxicos mostraron dificultades con el conocimiento ortográfico, a pesar de que cursaban el último nivel de educación media superior (bachillerato o doceavo grado), alcanzando un total de errores ortográficos en un instrumento estandarizado para su población que los ubicó por arriba del percentil 85. Adicionalmente, todos ellos tenían una velocidad lectora significativamente por debajo de lo esperado para su edad y grado académico; presentaron un promedio de velocidad lectora de 124 palabras por minuto, que de acuerdo con los estándares nacionales de habilidad lectora de México (SEP, 2010), equivale a quinto grado de educación primaria. El bajo conocimiento ortográfico, aunado a una lenta velocidad lectora, señalaron de manera clara la existencia de dificultades residuales en el aprendizaje de la lectura y por ello fueron clasificados como disléxicos, aún cuando muchos de ellos no eran conscientes de que sus dificultades forman parte de un trastorno de origen neurobiológico.

La falta de diagnóstico en la infancia, así como la ausencia de detección y atención de los jóvenes universitarios, es actualmente una preocupación en el área de la educación (Ibáñez et al., 2019; López-Escribano et al., 2018; Stampolitzis et al., 2017), no sólo en jóvenes hispanos, sino también en población inglesa, Hanley (1997) reportó que un grupo de jóvenes universitarios, que en su mayoría no tenían un diagnóstico previo, presentaban alteraciones significativas en lectura y ortografía en comparación con un grupo control. 


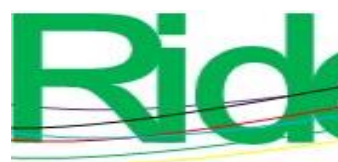

\section{Revista Iberoamericana para la Investigación y el Desarrollo Educativo ISSN $2007-7467$}

principales problemas que enfrentan los adultos con dislexia (Logan, 2009; Maughan et al., 2009; McLoughlin y Leather, 2013).

Se ha reportado que el puntaje total de los cuestionarios puede distinguir a los disléxicos de los que no los son, pero a diferencia de lo que reporta el propio autor del cuestionario aplicado (Vinegrad (1994), y los resultados de un estudio con estudiantes griegos en lo que se encontraron diferencias significativas en el puntaje total de un cuestionario de dislexia (Stampolitzis et al., 2017), en el presente estudio no se encontraron diferencias significativas entre los grupos en el puntaje total, probablemente derivado de la alta incidencia de dificultades reportadas por los jóvenes del grupo control para discriminar derecha-izquierda, para leer y orientarse en un mapa y para recordar lo que acaban de leer.

Consideramos que existen otras dificultades asociadas con la dislexia, que no son consideradas en las escalas y que se relacionan con aspectos más específicos de la lectura que merecerían ser exploradas, como puede ser la dificultad para leer material nuevo; para responder exámenes escritos; para expresar por escrito las ideas de manera clara al escribir cartas, reportes e incluso mensajes; además de dificultades con el uso de signos de puntuación. La exploración de aspectos más estrictamente relacionados con el proceso lector, podría ayudar a la identificación de estos jóvenes y a clarificar la magnitud de su trastorno, el cual puede poner en riesgo sus aspiraciones educativas, restringir su elección vocacional, llevarlos a abandonar su educación después del bachillerato (González-Garrido et al., 2014) e incluso conducirlos a desarrollar bajos niveles de autoeficacia y ansiedad en el trabajo, derivados de las emociones negativas emanadas de vivir con dislexia (Nalavany et al., 2017).

La dislexia tiene obvias repercusiones en la vida académica y laboral de las personas, pero se habla poco del impacto en la vida social y emocional resultante del choque constante con el desconocimiento e incomprensión de padres, maestros y autoridades educativas, quienes muchas veces los juzgan duramente por su lentitud para leer, por sus escritos incoherentes, plagados de errores ortográficos o por su marcada lentitud para responder exámenes y realizar cualquier escrito escolar, restándole importancia al resto de sus capacidades y a su potencial en diversas áreas. Las experiencias adversas de una infancia con dislexia pueden afectar negativamente en la adultez, determinando que muchos jóvenes y adultos presenten baja autoestima, ansiedad, depresión y estrés (Nalavany et al., 2010).

La falta de comprensión social de lo que la dislexia representa, aunada a los mitos existentes sobre ella, dificulta a los maestros y autoridades escolares comprender el enorme esfuerzo que representa para algunos jóvenes la lectura en voz alta, la escritura coherente y 


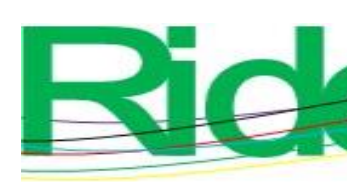

\section{Revista Iberoamericana para la Investigación y el Desarrollo Educativo ISSN $2007-7467$}

4) ¿Encuentras difícil y confuso llenar formas o solicitudes?

5) ¿Tienes problemas con la construcción de oraciones y con el uso de signos de puntuación?

6) ¿Te resulta más difícil que a tus compañeros escribir un reporte o un ensayo?

7) ¿Se te dificulta detectar los errores que has cometido en un escrito?

La validez de este breve cuestionario para identificar dificultades lectoras deberá ser analizada en estudios posteriores.

\section{Conclusiones}

La aplicación de la escala de Vinegrad a un gran número de jóvenes preuniversitarios, con la intención de identificar las principales dificultades que presentan aquellos clasificados como disléxicos (por su baja velocidad lectora y pobre conocimiento ortográfico), nos permitió confirmar que la mala ortografía, la dificultad para el llenado de formas y el desagrado por leer en voz alta son rasgos residuales que estos jóvenes reportan con mayor frecuencia en comparación con sus pares sin dificultades lectoras. Proponemos incluir estos tres aspectos, en conjunto con otras cuatro preguntas sobre problemas específicos de lectoescritura para integrar un cuestionario breve de identificación de dislexia en jóvenes y adolescentes, que requerirá ser validado con posterioridad.

Las escalas o cuestionarios de autoreporte no son pruebas rigurosas para el diagnóstico de dislexia, sin embargo, pueden brindar un excelente sondeo de rasgos de dificultades lectoras, que permita detectar rápidamente a aquéllos jóvenes que requieren de una valoración más amplia y un apoyo psicodidáctico especializado.

Los maestros, los padres y la sociedad en general, deben conocer las dificultades por las que pasan los disléxicos y comprender que los problemas lectores son una condición que va a acompañarlos toda su vida, independientemente del esfuerzo que se destine a su rehabilitación. Las autoridades educativas, por su parte, deben crear políticas para la detección temprana de estos casos, para el otorgamiento de apoyos y disminución de las barreras que obstaculizan el desarrollo académico en estos jóvenes. Los profesionales en educación, psicólogos y orientadores vocacionales deben recibir información actualizada, basada en evidencias científicas, sobre la naturaleza del trastorno y sus implicaciones educativas, personales y sociales, para poder brindar estrategias de tratamiento más apropiadas tanto a niños como a adolescentes. 


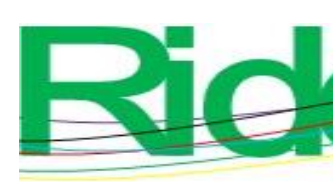

Revista Iberoamericana para la
Investigación y el Desarrollo Educativo
ISSN $2007-7467$

Futuras líneas de investigación

Explorar la validez y confiabilidad del cuestionario breve de 7 preguntas para exlorar las dificultades lectoras en jóvenes preuniversitarios, lo que permitiría la identificación temprana de aquéllos que enfrentan problemas residuales en el aprendizaje de la lectura.

\section{Fuentes de financiamiento}

Esta investigación se realizó dentro del proyecto 183561 subvencionado por el fondo mixto SEP-CONACYT del Gobierno de México, otorgado al primer autor.

\section{Referencias}

Decker, S. N., Vogler, G. P., y DeFries, J. C. (1989). Validity of Self-Reported Reading Disability by Parents of Reading-Disabled and Control Children. Reading and Writing: An Interdisciplinary Journal, 1(4), 327-331. https://doi.org/10.1007/BF00386265

De Luca, M., Di Pace, E., Judica, A., Spinelli, D., y Zoccolotti, P. (1999). Eye movement patterns in linguistic and non-linguistic tasks in developmental surface dislexia. Neuropsychologia, $\quad 37(12), \quad$ 1407-1420. $\quad$ https://doi.org/10.1016/S00283932(99)00038-X

Dyslexia Association of Ireland. "Adult Dyslexia Checklist". Recuperado de: https://www.dyslexia.ie/information/adults-and-the-workplace/adult-dyslexiachecklist/

Ehri, L. C. (2014). Orthographic mapping in the acquisition of sight word reading, spelling memory, and vocabulary learning. Scientific Studies of Reading, 18(1), 5-21. https://doi.org/10.1080/10888438.2013.819356

Gilger, J. W. (1992). Using self-report and parental- report survey data to assess past and present academic achievement of adults and children. Journal of Applied Developmental Psychology, 13(2), 235-256. https://doi.org/10.1016/01933973(92)90031-C

Giménez, A., Luque, J. L., López-Zamora, M., y Fernández-Navas, M. (2015). A self-report of reading disabilities for adults: ATLAS. Anales de Psicología, 31(1), 109-119. https://doi.org/10.6018/analesps.31.1.166671 


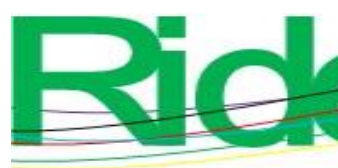

Revista Iberoamericana para la Investigación y el Desarrollo Educativo ISSN $2007-7467$

Gómez-Velázquez, F. R., González-Garrido, A. A., Guàrdia-Olmos, J., Peró-Cebollero, M., Zarabozo-Hurtado, D., y Zarabozo, D. (2014). Evaluación del conocimiento ortográfico en adultos jóvenes y su relación con la lectura. Revista Neuropsicología, Neuropsiquiatría y Neurociencias, 14(1), 40-67.

Gómez-Velázquez, F. R., González-Garrido, A. A., Zarabozo, D., y Amano, M. (2010). La velocidad de denominación de letras. El mejor predictor temprano del desarrollo lector en español. Revista mexicana de Investigación educativa, 15(46), 823-847.

González-Garrido, A. A., Gómez-Velázquez, F. R., y Rodríguez-Santillán, E. (2014). Orthographic recognition in late adolescents: An assessment through event-related brain potentials. Clinical EEG and Neuroscience, 45(2), 113-121. https://doi.org/10.1177/1550059413489975.

González-Garrido, A. A., Barrios, F. A., Gómez-Velázquez, F. R., y Zarabozo-Hurtado, D. (2017). The supramarginal and angular gyri underlie orthographic competence in a Spanish language. Brain and Language, 175, 1-10. https://doi.org/10.1016/j.bandl.2017.08.005

Hanley, J. R. (1997). Reading and spelling impairments in undergraduate students with developmental dislexia. Journal of Research in Reading, 20(1), 22-30. https://doi.org/10.1111/1467-9817.00017

Hebert, M., Zhang, X., y Parrila, R. (2018). Examining Reading comprehension text and question answering time differences in universitary students with and without a history of Reading difficulties. Annals of Dyslexia, 68(1), 15-24. https://doi.org/10.1007/s11881-017-0153-7

Ibáñez, A. E., Martin-Lobo, P., Vergara-Moragues, E., y Calvo, A. (2019). Profile and neuropsychological differences in adolescent students with and without dyslexia. Revista Latinoamericana de Psicología, 51(2), 83-92. http://dx.doi.org/10.14349/rlp.2019.v51.n2.4

Landerl, K., y Wimmer, H. (2008). Development of word reading fluency and spelling in a consistent orthography: An 8-year follow-up. Journal of Educational Psychology, 100(1), 150-161. https://doi.org/10.1037/0022-0663.100.1.150

Lefly, D. L., y Pennington, B. F. (2000). Reliability and validity of the Adult Reading History Questionnaire. Journal of Learning Disabilities, 33(3), 286-296. Available at https://dyslexiaida.org/screening-for-dyslexia/dyslexia-screener-for-adults/ 


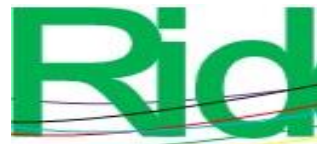

$+$

https://doi.org/10.11144/Javeriana.upsy15-2.adid

Stampoltzis, A., Tsitsou, E., Plesti, H., y Kalouri, R. (2017). Personal, educational and psychological characteristics of university students with dyslexia and matched controls: A pilot study. Innovative Practice in Higher Education, 3(1), 91-107.

Vellutino, F., y Fletcher, J. M. (2007). Developmental dyslexia. In M. J. Snowling \& C. Hulme (Eds.), The science of reading: A handbook (pp. 362-378). Oxford, England: Blackwell.

Vinegrad, M. (1994). A revised adult dyslexia checklist. Educare, 48, 21-23. Available at http://beta.dyslexiainternational.org/content/Checklists/DyslexiaCheckAdultsVinegr ad.pdf.

Wechsler, D. (2002). WAIS - III. Escala de inteligencia para adultos de Wechsler. Tercera edición. Buenos Aires: Paidós.

Wolff, U., y Lundberg, I. (2003). A technique for group screening of dyslexia among adults. Annals of Dyslexia, 53(1), 324-339. Available at www.jstor.org/stable/23764745. 


\begin{tabular}{|c|c|}
\hline Rol de Contribución & Autor (es) \\
\hline Conceptualización & Fabiola R. Gómez-Velázquez \\
\hline Metodología & Fabiola R. Gómez-Velázquez \\
\hline \multicolumn{2}{|l|}{ Software } \\
\hline Validación & $\begin{array}{l}\text { Fabiola R. Gómez-Velázquez (principal) } \\
\text { Jacobo J. Brofman-Epelbaum }\end{array}$ \\
\hline Análisis Formal & Fabiola R. Gómez-Velázquez \\
\hline Investigación & $\begin{array}{l}\text { Alicia Martínez-Ramos, Itzel Vergara, Jacobo J Brofman- } \\
\text { Epelbaum (igual contribución de los tres autores) }\end{array}$ \\
\hline Recursos & Fabiola R. Gómez-Velázquez \\
\hline \multicolumn{2}{|l|}{ Curación de datos } \\
\hline $\begin{array}{l}\text { Escritura - Preparación del } \\
\text { borrador original }\end{array}$ & Fabiola R. Gómez-Velázquez \\
\hline $\begin{array}{l}\text { Escritura - Revisión y } \\
\text { edición }\end{array}$ & Vanessa D. Ruiz-Stovel \\
\hline Visualización & $\begin{array}{l}\text { Fabiola R. Gómez-Velázquez (principal) } \\
\text { Itzel Vergara }\end{array}$ \\
\hline Supervisión & Fabiola R. Gómez-Velázquez \\
\hline Administración de Proyectos & $\begin{array}{l}\text { Fabiola R. Gómez-Velázquez } \\
\text { Alicia Martínez-Ramos }\end{array}$ \\
\hline Adquisición de fondos & Fabiola R. Gómez-Velázquez \\
\hline
\end{tabular}

